\title{
Development of a Safety- and Energy-Aware Impedance Controller for Collaborative Robots
}

\author{
Gennaro Raiola $^{(0)}$, Carlos Alberto Cardenas, Tadele Shiferaw Tadele ${ }^{(\mathbb{1})}$, Theo de Vries ${ }^{(\mathbb{})}$, and Stefano Stramigioli ${ }^{(\mathbb{1}}$
}

\begin{abstract}
In contexts where robots share their workspace with humans, safety is of utmost importance. Consequently, in recent years, a big impulse has been given to the design of human-friendly robots by involving both mechanical and control design aspects. Regarding controller design, this often involves introducing compliance and ensuring asymptotic stability using an interaction control scheme and passivity theory. Moreover, when human operators physically interact with the robot during work, strict safety measures become necessary with some of these including power and force limitations. In this letter, a novel impedance control technique for collaborative robots is presented. The featured controller allows a safe human-robot interaction through energy and power limitations, assuring passivity through energy tanks. The proposed controller is evaluated with a KUKA LWR $4+$ arm in a comanipulation environment.
\end{abstract}

Index Terms-Robot safety, physical human-robot interaction, compliance and impedance control.

\section{INTRODUCTION}

$\mathbf{R}$ ECENT advances in the robotics field have accelerated the development of robots that can operate close to humans. To emphasize this feature, these new type of robots are often called Cobots, from the neologism of the words collaborative and robot [1]. Since these robots are expected to share their workspace with a human operator, physical contact may occur for two reasons mainly: accidentally, in case of collisions; or deliberately, if the human operator is supposed to physically interact with the robot during work. In both cases, it is important to guarantee a safe physical interaction for injury prevention [2]. In this work, a novel impedance controller with safety limitations

Manuscript received September 9, 2017; accepted December 31, 2017. Date of publication January 23, 2018; date of current version February 8, 2018. This letter was recommended for publication by Editor Paolo Rocco upon evaluation of the Associate Editor and Reviewers' comments. This work was supported by the European Union's Horizon 2020 Research and Innovation Programme under Grant Agreement No.688857 (SoftPro). (Corresponding author: Gennaro Raiola.)

G. Raiola is with the Robotics and Mechatronics group, University of Twente, Enschede $7522 \mathrm{NH}$, The Netherlands, and also with the Department of Advanced Robotics, Istituto Italiano di Tecnologia, Genoa 16159, Italy (e-mail: gennaro.raiola@iit.it).

C. A. Cardenas, T. S. Tadele, T. de Vries, and S. Stramigioli are with the Robotics and Mechatronics group, University of Twente, Enschede 7522 NH, The Netherlands (e-mail: c.a.cardenasvilla@student.utwente.nl; t.s.tadele@utwente.nl; t.j.a.devries@utwente.nl; s.stramigioli@ieee.org ).

This letter has supplementary downloadable material available at http://ieeexplore.ieee.org, provided by the authors. The Supplemental Material contains a video. This material is $8 \mathrm{MB}$ in size.

Digital Object Identifier 10.1109/LRA.2018.2795639

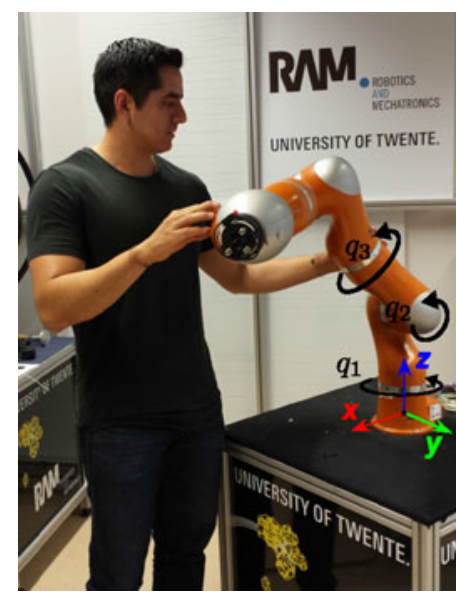

Fig. 1. Experimental setup with the KUKA-LWR 4+ robot.

is introduced based on the preliminary work presented in [3]. The work done in [3], introduces an impedance controller with safety limitations which is defined through a risk based safety analysis performed following the guideline defined in [4]. In particular, power and total energy of the system are considered as safety metrics for the analysis. The result is a variable impedance controller for a 1-DOF system which guarantees safety through energy and power limitation and establishes passivity and energy consistency of the system by incorporating an energy-tank as a tool for Passivity-Based Control (PBC) [5], [6].

In the present work, the impedance controller and the energy-tank notion from [3], are both extended and generalized from a single motor to a multi-DOF robot manipulator. Such generalization is made possible thanks to a geometric analysis performed with skrew theory. Moreover, the energy-tank conception is extended from a single to a multi tanks instance by taking in account problematics related to the tanks interconnection. Finally, these contributions are evaluated in a co-manipulation context with the KUKA LWR 4+ both in simulation and on the real system, see Fig. 1.

The rest of this letter is structured as follows. In the next section, related work is discussed. In Section III, the safetyaware impedance controller is presented, starting from the 1-DOF instance introduced in [3]. Passivity of the controller is discussed in Section IV. The approach is evaluated with a KUKA LWR 4+ manipulator, whose results are presented in Section V, ending with some final conclusions in Section VI. 


\section{RELATED WORK}

Collaborative robots are expected to operate in unstructured human environments and interact with unknown objects [7]. Thus, controller design should account for safety issues such as: introducing compliance to minimize injury in case of an uncontrolled impact, provide limitations in terms of velocities or forces exerted by the robot, and ensuring asymptotic stability even during interaction [8].

\section{A. Safety Criteria and Applications in Robotics}

While addressing the issue of human friendly robots, different safety criteria can be defined based on risk assessments [9]. For example, a power based safety metric called Head Impact Power (HIP) is proposed in [10]. In this work, the probabilities and the related power values of concussion from an impact on a human head are investigated and presented. Regarding energy criteria instead, the works presented in [11] and [12], identify the maximum allowed energy that can cause neck fracture and failure to cranial bones, respectively. One of the most widely used safety norms regarding robotic safety is the Head Injury Criteria (HIC), which was first proposed within the automotive industry by Versace in [13] and successively adopted in robotics [14]. This metric, along with the previously mentioned, have constituted an useful foundation for the development and evaluation of safety based robot controllers [15]-[17]. In [15], the $\mathrm{HIC}$ is used as a safety criteria to identify a performance limit, given in terms of maximum allowed link velocity. Hence, one of the control objectives is to guarantee that the desired trajectories satisfy a safety velocity limitation. In [16], the maximum impact force that can be exerted by a multi-DOF robot is used to define a force based safety metric, called impact potential. Regarding energy limitations as safety measures, [17] uses energy based metrics to design an energy regulation controller that limits the total energy of a robot within the required safety limit by modulating the desired trajectory reference. Similarly to [17], a controller which limits the energy exerted by the robot is defined in this letter; nevertheless, the energy limitation is applied directly through modulation of the controller's parameters instead of the reference trajectory.

\section{B. Stability Through Passivity}

Concerning the stability problem instead, different authors used passivity theories to design controllers which ensure asymptotic stability of the robot [18], [19]. By definition, passive systems are stable dynamic systems whose total energy is less than, or equal to, the sum of its initial energy and any external energy supplied to it by interaction [6]. As proven in [20], if the robot is not strictly passive, it is always possible that a passive environment destabilizes the robot motions and extracts infinite energy from it. Since most tasks can be defined in terms of energy, different authors introduced energy-tank methods to preserve passivity [21]-[23]. By using energy-tanks, the robot can use a certain amount of energy to perform a task, but no more than that. Different methodologies have been used to define such strategy. In this work, port-Hamiltonian systems theory is used

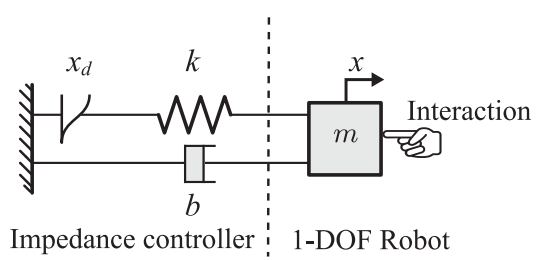

Fig. 2. Impedance controller connected to a mass representing a simple 1-DOF robot.

to define energy-tanks similarly to [21], giving them a physical interpretation to better understand the energy exchange between controller and robot. In general, the port-Hamiltonian formulation, gives a theoretic approach which allows to describe any physical system as a composition of multiple subsystems which are connected through ports. These ports can be used to track all energy flowing in the system. The advantage of such approach resides in the possibility of having systems that are fully Energy-Aware. For this reason, the port-Hamiltonian formulation provides an elegant and simple representation to describe energy-tanks and to study the passivity of systems. In literature, other techniques have been introduced for passivating systems through energy considerations. In [24] and successively in [25], the passivity observer (PO) and passivity controller (PC) are used to guarantee passive interaction with a haptic interface. To do so, the PO keeps track of the system's energy while the PC compensates eventual spurious energy generation by injecting damping. Differently, the presented methodology with energytanks defined through a port-Hamiltonian system, structurally prevents that any action taken would result in spurious energy generation, including the coupling between the discrete and continuous time, as proven in [26]. Indeed, if the energy in the controller is lower bounded, any control action is carried out in a way that will not generate energy. Therefore, energy can only be injected in the energy-tank externally (e.g., by the user) and from nowhere else [5].

The control scheme presented in this work, begins with a basic impedance controller whose parameters are first defined according to certain performance requirements [27]. These parameters (i.e., stiffness and damping), are then scaled such that safety limits defined in a combined energy and power based metrics are met. The resultant variable impedance controller is connected to an energy-tank subsystem to guarantee passivity and energy consistency of the overall system.

\section{SAFETY-AWARE IMPEDANCE CONTROL}

One of the advantages of using a Cartesian impedance controller [28] resides in its clear and simple physical interpretation. A Cartesian impedance controller can be seen as a mechanical spring-damper system connected to the robot's end-effector (Fig. 2). This design choice, allows a simple analysis of the controller's energy and power exchanged with the robot.

\section{A. Background Work: Safety-Aware Impedance Control for a 1-DOF System}

In the work presented in [3], the concept of a Safety-Aware Impedance Controller was introduced for a 1-DOF robot; an 
overview of this concept is introduced in this section as a fundamental starting point of the major contributions of this work. Consider a simplified robotic system represented by a single mass, controlled by an elemental impedance controller as shown in Fig. 2.

The total energy of the system is defined as the sum of the kinetic energy of the mass, and the potential energy of the spring:

$$
E_{\mathrm{tot}}=T_{k}+V_{p}=\frac{1}{2} m \dot{x}^{2}+\frac{1}{2} k x_{e}^{2}
$$

where $x_{e}=x_{d}-x$ is the motion error given a desired set-point $x_{d}, m$ is the mass of the simplified robot, and $\dot{x}$ represents its velocity. Given the total energy of the system (1), the objective is to design an impedance controller which is aware of the maximum amount of energy that a human can tolerate without sustaining injury; this is attainable by designing a controller that limits the energy interchanged with the human operator by adjusting the stiffness $k$ with respect to a maximum allowed energy which is delimited by $E_{\max }$ :

$$
k= \begin{cases}k_{0} & E_{\text {tot }} \leq E_{\max }, \\ \frac{2 E_{\max }-m \dot{x}^{2}}{\left(x_{d}-x\right)^{2}} & E_{\text {tot }}>E_{\max },\end{cases}
$$

where $k_{o}$ is the maximum controller stiffness which is chosen based on performance requirements. Once the safe energy restrictions are assured by stiffness adjustment, the maximum power that the robot can transfer to a human, during interaction, can be limited by increasing the dissipative damping. Given the impedance characteristic of the controller, the power $P_{c}$ flowing from it to the robot can be written as:

$$
P_{c}=\left(k\left(x_{d}-x\right)-b \dot{x}\right) \dot{x} .
$$

Therefore, for a fixed controller stiffness value $k$, the power can be limited to a maximum value $P_{\max }$ by defining the damping $b$ as follows:

$$
b= \begin{cases}b_{0} & P_{c} \leq P_{\max }, \\ \frac{k\left(x_{d}-x\right) \dot{x}-P_{\max }}{\dot{x}^{2}} & P_{c}>P_{\max } .\end{cases}
$$

Once the stiffness and damping parameters are determined with (2) and (4), respectively, the impedance control law is finally defined as:

$$
F_{c}=k\left(x_{d}-x\right)-b \dot{x} .
$$

The overall effect of the chosen control is to enforce a compliant behavior when the energy limits are violated, and to decelerate the robot's motion if the power limits are exceeded. Moreover, since the two limitations are activated simultaneously, the lower limitation could affect the activation of the other safety limit. For example, by imposing a low power limit, the system would be strongly damped and so, depending on the reference, the tracking error could produce a high spring energy causing the energy limit to be activated.

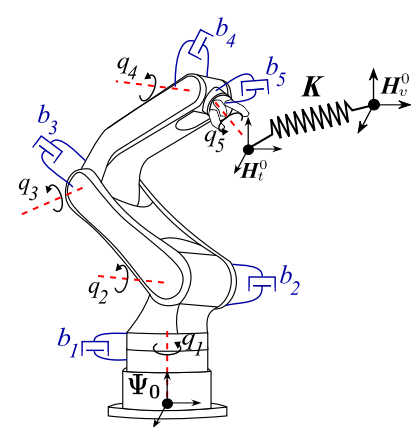

Fig. 3. Cartesian impedance control of a robot. A multidimensional virtual spring is connected between the current, and desired end-effector's configuration and damping is added at each joint.

\section{B. Contribution: Safety-Aware Impedance Control for a Multi-DOF Robot}

In this section, the safety-aware impedance control is extended to a multi-DOF robotic manipulator, which is one of the main contributions of this work. The Lagrangian dynamic equation for a rigid multi-DOF robot is given as:

$$
\boldsymbol{M}(\boldsymbol{q}) \ddot{\boldsymbol{q}}+\boldsymbol{C}(\boldsymbol{q}, \dot{\boldsymbol{q}}) \dot{\boldsymbol{q}}+\boldsymbol{G}(\boldsymbol{q})=\boldsymbol{\tau},
$$

where $\boldsymbol{M}(\boldsymbol{q})$ is the inertia matrix, $\boldsymbol{C}(\boldsymbol{q}, \dot{\boldsymbol{q}})$ represents the Coriolis and centrifugal terms, $\boldsymbol{G}(\boldsymbol{q})$ is the gravitational term, and $\tau$ represents the torques acting on the joints given by the controller action and the external interaction. Given two homogeneous matrices $\boldsymbol{H}_{t}^{0}$ and $\boldsymbol{H}_{v}^{0}$, describing the current and desired end-effector's configuration, respectively, the Cartesian impedance controller for a multi-DOF robot is physically described by a multidimensional spring with symmetric stiffness matrix $\boldsymbol{K} \in \mathbb{R}^{6 \times 6}$, attempting to co-align these configurations. In order to achieve a satisfactory dynamic behavior, and guarantee robust asymptotic stability, damping is added to the system. Damping can be injected either in Cartesian space via a multidimensional damper matrix $\boldsymbol{B} \in \mathbb{R}^{6 \times 6}$, or in joint space via a damper $b_{n}$ on each DOF. In this work, the latter is taken into consideration, see Fig. 3. With these considerations, the wrench applied on the manipulator due to the virtual spring connection is defined as:

$$
\boldsymbol{W}^{t}=\left(\begin{array}{c}
\boldsymbol{m}^{t} \\
\boldsymbol{f}^{t}
\end{array}\right)=\left(\begin{array}{cc}
\boldsymbol{K}_{o} & \boldsymbol{K}_{c} \\
\boldsymbol{K}_{c}^{\top} & \boldsymbol{K}_{t}
\end{array}\right)\left(\begin{array}{c}
\delta \boldsymbol{\theta}_{t}^{v} \\
\delta \boldsymbol{p}_{t}^{v}
\end{array}\right),
$$

where $\delta \boldsymbol{T}=\left[\left(\delta \boldsymbol{\theta}_{t}^{v}\right)^{\top}\left(\delta \boldsymbol{p}_{t}^{v}\right)^{\top}\right]$ is an infinitesimal twist in vector form and $\boldsymbol{K}_{t}, \boldsymbol{K}_{o}$, and $\boldsymbol{K}_{c}$, are the symmetric translational, rotational and coupling stiffness matrices. Given the components of the stiffness matrix, it is possible to define the respective co-stiffness matrices $\boldsymbol{G}_{t}, \boldsymbol{G}_{o}$ and $\boldsymbol{G}_{c}$ as

$$
\boldsymbol{G}_{x}=\frac{1}{2} \operatorname{tr}\left(\boldsymbol{K}_{x}\right) I-\boldsymbol{K}_{x},
$$

where $\operatorname{tr}()$ is the tensor trace operator. The generalized wrench exerted by the spring is a function of the relative configuration $\boldsymbol{H}_{t}^{v}$, which can be defined as

$$
\boldsymbol{H}_{t}^{v}=\left(\boldsymbol{H}_{0}^{v}\right)^{-1} \cdot \boldsymbol{H}_{t}^{0}=\left(\begin{array}{cc}
\boldsymbol{R}_{t}^{v} & \boldsymbol{p}_{t}^{v} \\
\mathbf{0}^{\top} & 1
\end{array}\right),
$$


and the wrench $\boldsymbol{W}^{t}=\left[\boldsymbol{m}^{t} \boldsymbol{f}^{t}\right]^{\top}$ exerted on the manipulator due to the spring is expressed in the coordinates of the end-effector frame, and can be rewritten as [29]:

$$
\begin{array}{r}
\tilde{\boldsymbol{m}}^{t}=-2 \operatorname{as}\left(\boldsymbol{G}_{o} \boldsymbol{R}_{t}^{v}\right)-\operatorname{as}\left(\boldsymbol{G}_{t} \boldsymbol{R}_{v}^{t} \tilde{\boldsymbol{p}}_{t}^{v} \tilde{\boldsymbol{p}}_{t}^{v} \boldsymbol{R}_{t}^{v}\right)-2 \operatorname{as}\left(\boldsymbol{G}_{c} \tilde{\boldsymbol{p}}_{t}^{v} \boldsymbol{R}_{t}^{v}\right), \\
\tilde{\boldsymbol{f}}^{t}=-\boldsymbol{R}_{v}^{t} \mathrm{as}\left(\boldsymbol{G}_{t} \tilde{\boldsymbol{p}}_{t}^{v}\right) \boldsymbol{R}_{t}^{v}-\operatorname{as}\left(\boldsymbol{G}_{t} \boldsymbol{R}_{v}^{t} \tilde{\boldsymbol{p}}_{t}^{v} \boldsymbol{R}_{t}^{v}\right)-2 \operatorname{as}\left(\boldsymbol{G}_{c} \boldsymbol{R}_{t}^{v}\right),
\end{array}
$$

where as() is an operator that gives the skew-symmetric part of a square matrix. After coordinate transformation of the wrench to an inertial reference frame $\Psi_{0}$, it is possible to transform the wrench into command torques $\tau_{c}$ for the robot by using the Jacobian transpose:

$$
\begin{aligned}
\left(\boldsymbol{W}^{0}\right)^{\top} & =A d_{\boldsymbol{H}_{0}^{t}}^{\top}\left(\boldsymbol{W}^{t}\right)^{\top}, \\
\boldsymbol{\tau}_{\boldsymbol{c}} & =\boldsymbol{J}^{\top}(\boldsymbol{q}) \boldsymbol{W}^{0},
\end{aligned}
$$

where $A d()$ is Adjoint of the homogeneous matrix. The dynamics of the robotic manipulator can be further shaped by adding a damping term $\bar{B}$, acting on the joints, and the gravity compensation term $\hat{\boldsymbol{G}}(\boldsymbol{q})$ in the command torques:

$$
\tau_{c}=\boldsymbol{J}^{\top}(\boldsymbol{q}) \boldsymbol{W}^{0}-\overline{\boldsymbol{B}} \dot{\boldsymbol{q}}+\hat{\boldsymbol{G}}(\boldsymbol{q}) .
$$

The resulting multidimensional Cartesian impedance controller (14) can be integrated with the safety metrics presented in Section III-A. In this case, it is first necessary to generalize the total energy of the system (1) to a multi-DOF instance. The kinetic energy for a multi-DOF robotic system is defined as:

$$
T_{k}(\boldsymbol{q}, \dot{\boldsymbol{q}})=\frac{1}{2} \dot{\boldsymbol{q}}^{\top} \boldsymbol{M}(\boldsymbol{q}) \dot{\boldsymbol{q}},
$$

while the potential energy of the spatial spring is given by:

$$
V_{p}\left(\boldsymbol{R}_{t}^{v}, \boldsymbol{p}_{t}^{v}\right)=V_{t}\left(\boldsymbol{R}_{t}^{v}, \boldsymbol{p}_{t}^{v}\right)+V_{o}\left(\boldsymbol{R}_{t}^{v}\right)+V_{c}\left(\boldsymbol{R}_{t}^{v}, \boldsymbol{p}_{t}^{v}\right),
$$

where $V_{t}\left(\boldsymbol{R}_{t}^{v}, \boldsymbol{p}_{t}^{v}\right), V_{o}\left(\boldsymbol{R}_{t}^{v}\right)$, and $V_{c}\left(\boldsymbol{R}_{t}^{v}, \boldsymbol{p}_{t}^{v}\right)$, represent the translational, rotational, and coupling elements of the potential energy which are computed as [29]:

$$
\begin{aligned}
V_{t}\left(\boldsymbol{R}_{t}^{v}, \boldsymbol{p}_{t}^{v}\right)= & -\frac{1}{4} \operatorname{tr}\left(\tilde{\boldsymbol{p}}_{t}^{v} \boldsymbol{G}_{t} \tilde{\boldsymbol{p}}_{t}^{v}\right)-\frac{1}{4} \operatorname{tr}\left(\tilde{\boldsymbol{p}}_{t}^{v} \boldsymbol{R}_{t}^{v} \boldsymbol{G}_{t} \boldsymbol{R}_{v}^{t} \tilde{\boldsymbol{p}}_{t}^{v}\right), \\
& V_{o}\left(\boldsymbol{R}_{t}^{v}\right)=-\operatorname{tr}\left(\boldsymbol{G}_{\mathrm{o}} \boldsymbol{R}_{\mathrm{t}}^{v}\right), \\
& V_{c}\left(\boldsymbol{R}_{t}^{v}, \boldsymbol{p}_{t}^{v}\right)=\operatorname{tr}\left(\boldsymbol{G}_{c} \boldsymbol{R}_{v}^{t} \tilde{\boldsymbol{p}}_{t}^{v}\right),
\end{aligned}
$$

with $\boldsymbol{G}_{t}, \boldsymbol{G}_{o}$, and $\boldsymbol{G}_{c}$ defined through (8).

Analogously to the 1-DOF case, energy limitation is established by regulating the amount of potential energy that the spatial spring supplies to the system. As it can be observed, the potential energy components in (17) are all proportional to the co-stiffness matrices $\boldsymbol{G}_{x}$ (for $x=t, o, c$ ). Consequently, by choosing a set of initial co-stiffness matrices $\boldsymbol{G}_{x_{i}}$, an initial potential energy value is defined, and the total energy of the system can be regulated by scaling the co-stiffness matrices with a scaling parameter $\lambda$ :

$$
\boldsymbol{G}_{x}=\lambda \cdot \boldsymbol{G}_{x_{i}} \quad \text { for } \quad x=t, o, c .
$$

Similarly to (2), the total energy is limited to a maximum allowed value $E_{\max }$ by selecting the scaling parameter $\lambda$ as:

$$
\lambda= \begin{cases}1 & E_{\text {tot }} \leq E_{\max }, \\ \frac{E_{\max }-T_{k}(\boldsymbol{q}, \dot{\boldsymbol{q}})}{V_{p_{i}}\left(\boldsymbol{R}_{t}^{v}, \boldsymbol{p}_{t}^{v}\right)} & \text { otherwise, }\end{cases}
$$

where $E_{\text {tot }}$, and $V_{p_{i}}\left(\boldsymbol{R}_{t}^{v}, \boldsymbol{p}_{t}^{v}\right)$ are the total and potential energies, respectively, due to the initial co-stiffness matrices $\boldsymbol{G}_{x_{i}}$. Given (19), the total energy of the system can be expressed as:

$$
E_{\mathrm{tot}}=T_{k}(\boldsymbol{q}, \dot{\boldsymbol{q}})+\lambda V_{p_{i}}\left(\boldsymbol{R}_{t}^{v}, \boldsymbol{p}_{t}^{v}\right) .
$$

By scaling the initial potential energy by $\lambda$, the total energy will always be less or equal than the maximum allowed energy value $E_{\max }$. After computing the new co-stiffness value (18), the power transferred from the controller to the robot can be regulated by adjusting the damping analogously to the 1-DOF case (4). If an initial damping matrix $\overline{\boldsymbol{B}}_{i}$ is chosen, the power transferred from the controller is given by the following expression:

$$
P_{c_{t o t}}=\underbrace{\left(\boldsymbol{J}^{\top}(\boldsymbol{q}) \boldsymbol{W}^{0}-\overline{\boldsymbol{B}}_{i} \dot{\boldsymbol{q}}\right)^{\top} \dot{\boldsymbol{q}}}_{P_{c}}+\underbrace{\hat{\boldsymbol{G}}(\boldsymbol{q})^{\top} \dot{\boldsymbol{q}}}_{P_{c_{g}}},
$$

where $\boldsymbol{W}^{0}$ is the wrench acting on the end-effector due to the spatial Cartesian spring, which is scaled by (19). In case of an uncontrolled collision with a human operator, $P_{c}$ represents the power that can be transferred to the operator since the power related to the gravity term $P_{c_{q}}$ gets dissipated by the robot itself to compensate its weight, and thus, $P_{c}$ has to be limited below a tolerance value $P_{\max }$. The latter is attained by defining a scaling parameter $\beta$ as:

$$
\beta= \begin{cases}1 & P_{c} \leq P_{\max }, \\ \frac{\left(\boldsymbol{J}^{\top}(\boldsymbol{q}) \boldsymbol{W}^{0}\right)^{\top} \dot{\boldsymbol{q}}-P_{\max }}{\dot{\boldsymbol{q}}^{\top} \overline{\boldsymbol{B}}_{i} \dot{\boldsymbol{q}}} & \text { otherwise. }\end{cases}
$$

Finally, the damping can be defined as:

$$
\overline{\boldsymbol{B}}=\beta \cdot \overline{\boldsymbol{B}}_{i} .
$$

\section{Ascertaining PASsivity Through ENERGy-TANKS}

Passive systems are a class of dynamical systems in which energy exchange with the environment plays a central role, since they cannot deliver more energy than what is stored. The connection between the energetic features of a system and its stability, is delineated by means of PBC [6]. The implementation of the safety-aware controllers presented in the previous sections contradicts the energetic consistency of impedance control design. The scaling of the parameters in the controller, allows internal energy production, resulting in the loss of passivity of the overall system. Therefore, the energy-tank based controller implementation presented in [3] for a 1-DOF case, is extended and generalized to a multi-DOF instance, in order to circumvent this issue. As discussed in Section II, energy-tank systems can be described through the port-Hamiltonian formulation. In general, energy-tanks are presented as tools that can be added to any task oriented controller, in order to guarantee stability in non passive environments. The latter, together with the controller presented in Section III-B, builds up the so-called Safety 


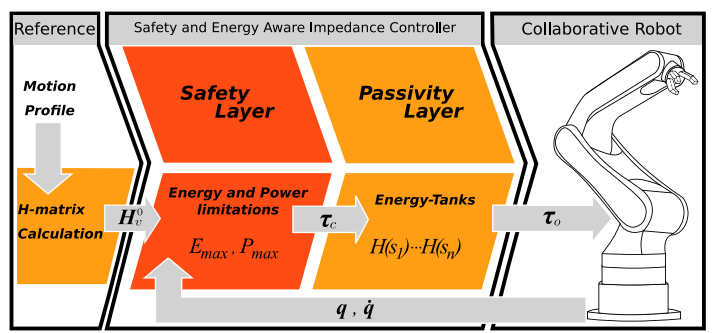

Fig. 4. Layered scheme of the proposed controller. The overall controller consists of two main layers: the safety layer which enforces the energy and power limitations as presented in Section III-B, and a passivity layer realized through energy-tanks. The motion profile can be generated either in Cartesian or joint space.

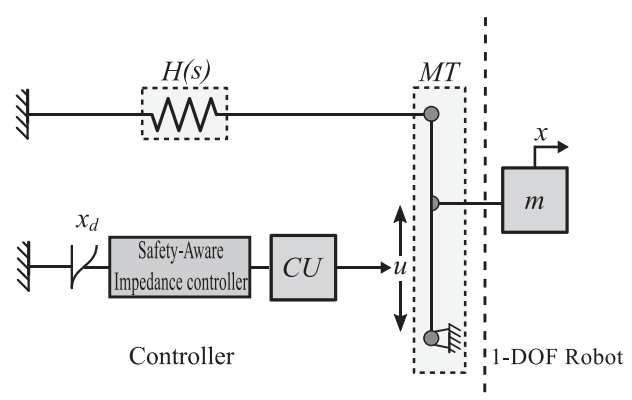

Fig. 5. 1-DOF energy-tank based controller.

and Energy Aware Impedance Controller, which is the major contribution of this work. This conception can be summarized in a multi-layered structure control strategy, illustrated in Fig. 4.

\section{A. Energy-Tank Based Controller for a 1-DOF System}

The physical representation of the energy-tank based controller for a 1-DOF system is depicted in Fig. 5. The energytank $H(s)$ is modeled as a spring with constant stiffness (e.g., $k=1$ ) connected to the robot through a transmission $M T$. This transmission allows power to flow from the controller to the robot, regulated by the ratio $u$, whose value is determined by a computational unit $C U$.

The port-Hamiltonian equation of the energy-tank system, can be expressed as:

$$
\left(\begin{array}{c}
\dot{s} \\
\dot{p}
\end{array}\right)=\left(\begin{array}{rr}
0 & u \\
-u & 0
\end{array}\right)\left(\begin{array}{c}
s \\
\dot{x}
\end{array}\right),
$$

where $p$ is the momentum of the robot, $\dot{x}$ is the velocity of the robot, and $s$ is the state of the spring. Given the controller output $F_{c}$ from (5), the port-Hamiltonian equation in (24) can be used to set the desired transmission ratio $u$ as:

$$
u=\frac{-F_{c}}{s} .
$$

The computational unit $C U$ assures that the power flows from the controller to the robot only if there is energy left in the tank. Hence, when the tank is empty, the controller's power output must be zero; in this way, passivity is guaranteed. Moreover, when power "flows back" to the controller, the energy-tank can be refilled with the injected energy. Given these considerations,

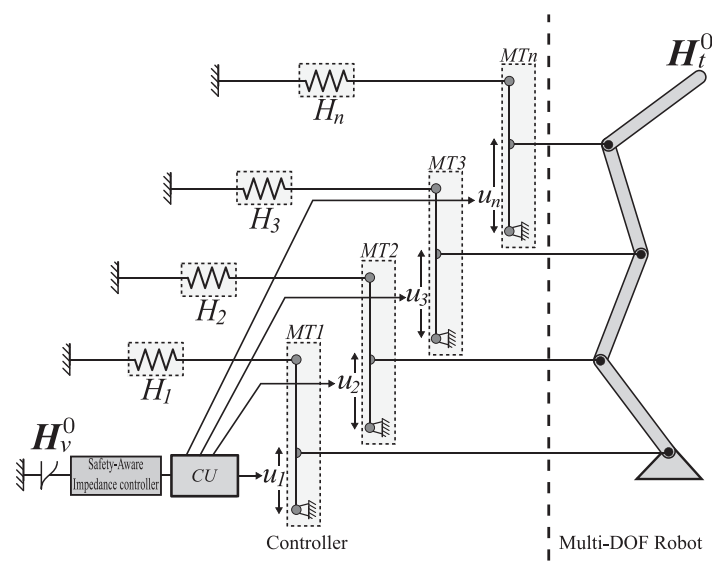

Fig. 6. Physical depiction of the energy-tank based controller for a multi-DOF robot.

$C U$ can be defined as a simple state machine:

$$
u= \begin{cases}\frac{-F_{c}}{s} & \text { if } \left.(H(s)>\epsilon) \vee\left(P_{c}<0\right)\right), \\ 0 & \text { otherwise, }\end{cases}
$$

where $H(s)=\frac{1}{2} k s^{2}$ is the potential energy in the tank and $\epsilon$ is the minimum amount of energy in the tank before the robot and the controller are decoupled. The state of the spring in $H(s)$ is computed at each time step by integrating $\dot{s}$ from the portHamiltonian expression in (24), adjusting in this way the value of the transmission ratio $u$. Note that the condition in (26) allows the computation of $u$, only when there is energy left in the tank, or when the power flowing from the controller to the robot is negative. The latter condition is satisfied when the tank is being recharged, which will only happen if an external force is applied to the system adding extra energy to the tank.

\section{B. Extension to a Multi-DOF Robot}

The general 1-DOF simplified case can be extended to a multi-DOF instance, where each joint is examined as the energy storage phenomenon illustrated in Fig. 5. The physical perception of the multi-DOF energy-tank notion is depicted in Fig. 6.

Analogously to the 1-DOF case, the port-Hamiltonian formulation for a joint subsystem $n$ can be disclosed as:

$$
\left(\begin{array}{c}
\dot{s}_{n} \\
\tau_{o_{n}}
\end{array}\right)=\left(\begin{array}{rr}
0 & u_{n} \\
-u_{n} & 0
\end{array}\right)\left(\begin{array}{l}
s_{n} \\
\dot{q}_{n}
\end{array}\right) .
$$

whereas in this case, the transmission ratio $u_{n}$ is determined as:

$$
u_{n}= \begin{cases}\frac{-\tau_{c_{n}}}{s_{n}} & \text { if }\left(H_{n}\left(s_{n}\right)>\epsilon\right), \\ \frac{-\tau_{c_{n}}}{\gamma^{2}} s_{n} & \text { otherwise, }\end{cases}
$$

where $\gamma=\sqrt{2 \cdot \epsilon}$, and $\tau_{c_{n}}$ is the torque computed with the chosen control strategy. Unlike (26), the piecewise function in (28) does not assign a value of zero to the transmission ratio when the energy-tank is depleted, such that a control action still takes place during the depleted state of the tank. This strategy circumvents the case where joint $\mathrm{n}$ extends its motion when 


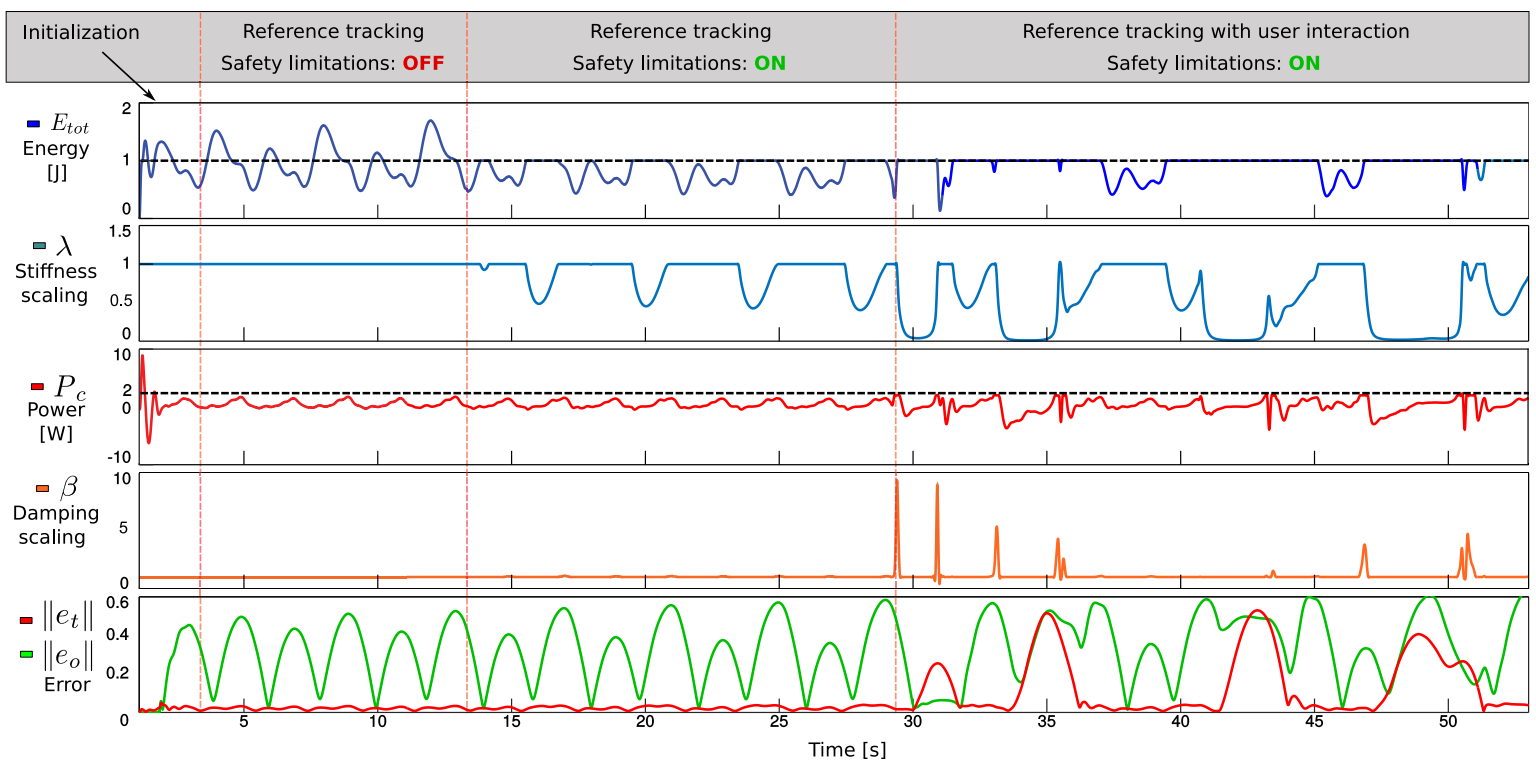

Fig. 7. The experiment shows the limitations in term of energy and power as well as the performances in terms of tracking error. In the first part of the experiment the safety limitations are not applied, the robot is able to track better the reference $\left\|e_{t}\right\|:(M=0.0220, S D=0.0076),\left\|e_{o}\right\|:(M=0.2790, S D=0.1619)$. In the second part the safety limits are active, thus the energy and power are limited to the their maximum values $\left(E_{\max }=1.0, P_{\mathrm{max}}=2.0\right)$ thanks to the scaling stiffness (19) and damping (22). As a consequence, the tracking error increases $\left\|e_{t}\right\|:(M=0.0293, S D=0.084),\left\|e_{o}\right\|:(M=0.2985, S D=0.1548)$. In the last part of the experiment, the user interacts with the robot while the safety limits are active thus the energy and power limitations guarantee a safe interaction $\left\|e_{t}\right\|:(M=0.0795, S D=0.1143),\left\|e_{o}\right\|:(M=0.3216, S D=0.1645)$.

$H_{n}\left(s_{n}\right) \leq \epsilon$ due to the inertial motion of the other links, which may unintentionally recharge the tank in case. Moreover, the power condition in the upper statement is removed from the if condition to avoid recharging the tanks due to the presence of noise in the computation of $P_{c_{n}}$. These are obliged adaptations for the multi-DOF instance, that induce passivity preservation of the overall system.

Once the transmission ratio is settled according to the energy levels in $H_{n}\left(s_{n}\right)$, the torque output sent to joint $n$ is computed as:

$$
\tau_{o_{n}}=-u_{n} \cdot s_{n}
$$

\section{EXPERIMENTAL RESULTS}

To validate the proposed control scheme, two experiments were conducted on the real platform and in simulation, using the KUKA LWR 4+ Fast Research Interface (FRI) [30] embedded in a ROS controller [31]. For both experiments, a Cartesian reference trajectory was defined as a periodic motion along the $y$-axis of the base frame $(T=4[\mathrm{~s}])$ :

$$
H_{v}^{0}(t)=\left\{\begin{array}{l}
p_{v}^{0}(t)=\left[\begin{array}{c}
x \\
y(t) \\
z
\end{array}\right]=\left[\begin{array}{c}
-0.6 \\
0.3 \sin \left(\frac{2 \pi}{T} t\right) \\
0.6
\end{array}\right], \\
R_{v}^{0}=\left[\begin{array}{rrr}
0 & 0 & -1 \\
0 & 1 & 0 \\
1 & 0 & 0
\end{array}\right] .
\end{array}\right.
$$

For the experiments the controller gains are defined as $\boldsymbol{K}_{t}=$ $1000 \boldsymbol{I}_{3}, \boldsymbol{K}_{o}=100 \boldsymbol{I}_{3}, \boldsymbol{K}_{c}=\mathbf{0}_{3}, \bar{B}=50 \boldsymbol{I}_{7}$, and the safety parameters as $\left(E_{\max }=1.0, P_{\max }=2.0\right)$.

\section{A. Experiment 1: Safe Human-Robot Interaction}

In order to evaluate the safety-aware controller and its performances while the user interacts with the robot, the experiment is divided in three parts. In the first part the safety limitations are not activated. In the second part, the controller enforces the limitations reducing both energy and power below the selected thresholds thanks to the scaling stiffness and damping. In the third part, the user interacts with the robot by constantly applying a force in different directions on the robot's end-effector, while safety limitations are enabled ${ }^{1}$. The tracking error for the translation part $\left\|e_{t}\right\|$ is computed as the norm of the difference vector $\boldsymbol{p}_{t}^{v}$, while to compute the orientation error from the rotation matrix $\boldsymbol{R}_{t}^{v}$, the angle and axis representation [32] is used. The results of the experiment as well as its description are presented in Fig. 7 to ease the reading.

\section{B. Experiment 2: Energetic Behavior of the Tanks}

The behavior of the energy-tank notion, under the presence of an external force, is examined in the following simulated experiment in conjunction with the safety layer. In particular, the experiment focuses on the interaction between the controller power and the energy-tanks.

Fig. 8 shows the controller power, scaling damping, and the relevant data from the first three joints, which are the joints primarily used by the manipulator for the given motion reference (30). A constant force $F_{e x t}=-10 \mathrm{~N}$ is applied on the robot's tool-tip for one second at $t=15[\mathrm{~s}]$ along the $y$-axis. Consequently, the energy tanks level increases due to the external force. Moreover, the tanks get refilled when the power becomes

\footnotetext{
${ }^{1}$ For a better understanding of the experiment we recommend viewing the video supplement.
} 


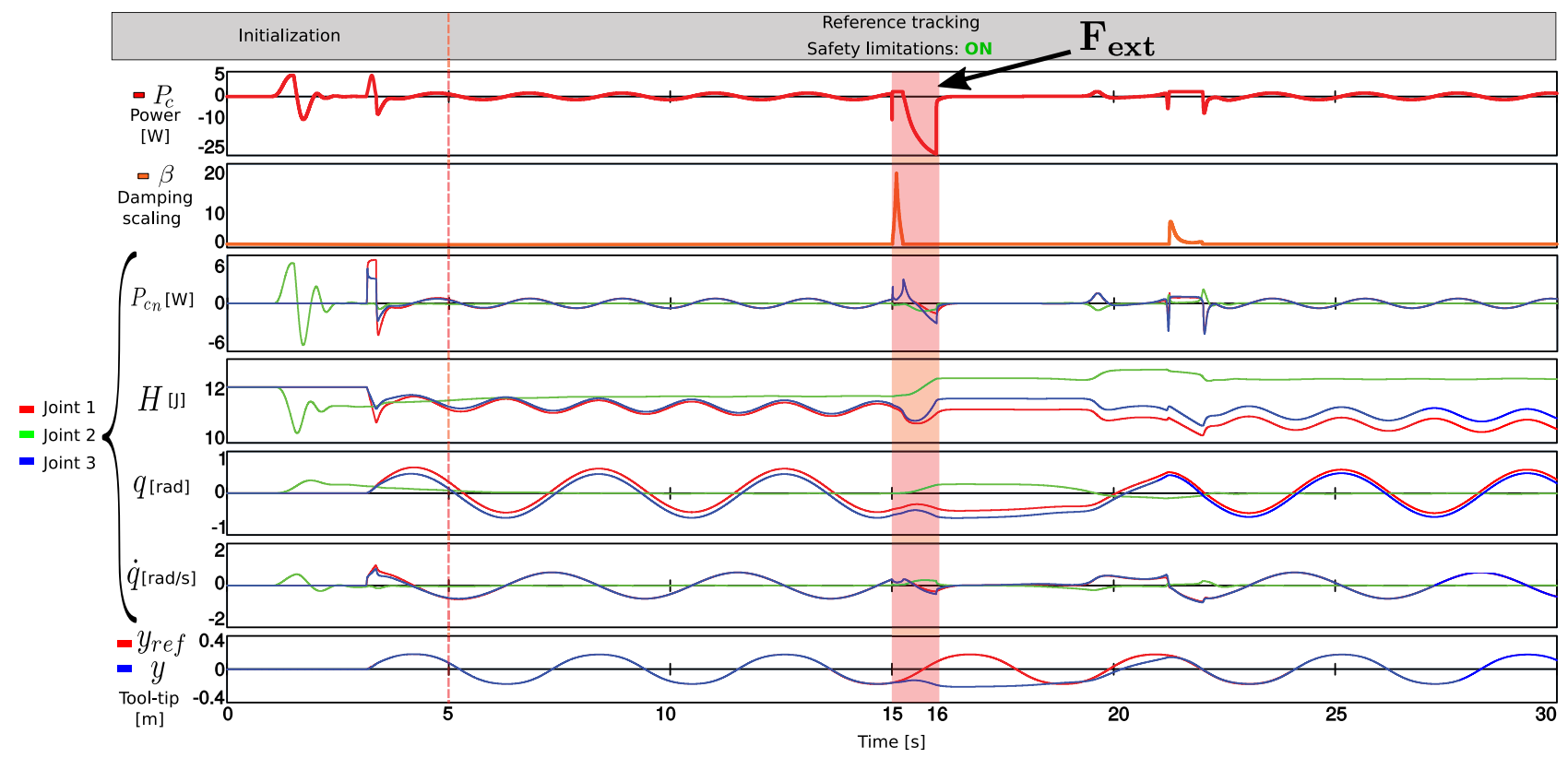

Fig. 8. After an initialization phase in which the robot starts tracking the reference, the safety limitations are activated. A constant force is applied for one second starting from $t=15 \mathrm{~s}$ (red area). Consequentially the tracking on the $y$-axis gets disrupted as shown in the last plot. The energy levels of the tanks are shown in the fourth plot. At the beginning of the experiment, their level is initialized at $12[\mathrm{~J}]$ and successively, slowly decreases as the energy gets used by the controller (joint 1 and 3). It is worth noting that joint 2 is not relevant for the chosen motion (its velocity is close to zero) and so it does not consume energy from its tank.

negative. This is a direct consequence of the increased damping value acting on the joints (23) and is coherent with the desired behavior expressed by (28).

\section{CONCLUSION}

A safety-aware impedance controller for multi-DOF collaborative robots is proposed. This controller ensures safety thanks to energy and power limitations, which are enforced by scaling accordingly stiffness and damping of the controller (Fig. 7). The passivity of the controller is assured by decoupling the controller from the robot through energy-tanks. Energy-tanks provide an easy and elegant solution for the passivity problem but their presence limits the system autonomy by introducing a maximum quantity of consumable energy after which the controller gets decoupled from the robot. For this reason, in future work, it will be investigated how to make an energy-tank system which is aware of the energy amount needed to perform a specific task, resulting in a more efficient energy-aware controller.

\section{REFERENCES}

[1] J. E. Colgate, M. A. Peshkin, and S. H. Klostermeyer, "Intelligent assist devices in industrial applications: A review," in Proc. IEEE/RSJ Int. Conf. Intell. Robots Syst., 2003, pp. 2516-2521.

[2] A. Bicchi, M. A. Peshkin, and J. E. Colgate, "Safety for physical humanrobot interaction," in Springer Handbook of Robotics. Berlin, Germany: Springer, 2008, pp. 1335-1348.

[3] T. S. Tadele, T. J. de Vries, and S. Stramigioli, "Combining energy and power based safety metrics in controller design for domestic robots," in Proc. 2014 IEEE Int. Conf. Robot. Autom., 2014, pp. 1209-1214.

[4] C. Harper and G. Virk, "Towards the development of international safety standards for human robot interaction," Int. J. Social Robot., vol. 2, no. 3, pp. 229-234, 2010.

[5] G. A. Folkertsma et al., "Energy in robotics," Found. Trends Robot., vol. 6, no. 3, pp. 140-210, 2017.
[6] R. Ortega, J. A. L. Perez, P. J. Nicklasson, and H. Sira-Ramirez, PassivityBased Control of Euler-Lagrange Systems: Mechanical, Electrical and Electromechanical Applications. Berlin, Germany: Springer Science \& Business Media, 2013.

[7] R. Alami et al., "Safe and dependable physical human-robot interaction in anthropic domains: State of the art and challenges," in Proc. 2006 IEEE/RSJ Int. Conf. Intell. Robots Syst., 2006, pp. 1-16.

[8] T. S. Tadele, T. de Vries, and S. Stramigioli, "The safety of domestic robotics: A survey of various safety-related publications," IEEE Robot. Autom. Mag., vol. 21, no. 3, pp. 134-142, Sep. 2014.

[9] M. Wassink and S. Stramigioli, "Towards a novel safety norm for domestic robotics," in Proc. IEEE/RSJ Int. Conf. Intell. Robots Syst., 2007, pp. 3354-3359.

[10] J. A. Newman, N. Shewchenko, and E. Welbourne, "A proposed new biomechanical head injury assessment function-the maximum power index." Stapp Car Crash J., vol. 44, pp. 215-247, 2000.

[11] J. L. Wood, "Dynamic response of human cranial bone," J. Biomech., vol. 4, no. 1, pp. 1IN13-2IN312, 1971.

[12] N. Yoganandan, F. Pintar, D. Maiman, J. Cusick, A. Sances, and P. Walsh, "Human head-neck biomechanics under axial tension," Med. Eng. Phys., vol. 18, no. 4, pp. 289-294, 1996.

[13] J. Versace, "A review of the severity index," SAE, Warrendale, PA, USA, Tech. Rep. 710881, 1971.

[14] S. Haddadin, A. Albu-Schäffer, and G. Hirzinger, "Safety analysis for a human-friendly manipulator," Int. J. Social Robot., vol. 2, no. 3, pp. 235$252,2010$.

[15] A. Bicchi and G. Tonietti, "Fast and "soft-arm" tactics [robot arm design]," IEEE Robot. Autom. Mag., vol. 11, no. 2, pp. 22-33, Jun. 2004.

[16] J. Heinzmann and A. Zelinsky, "Quantitative safety guarantees for physical human-robot interaction," Int. J. Robot. Res., vol. 22, no. 7-8, pp. 479-504, 2003.

[17] M. Laffranchi, N. G. Tsagarakis, and D. G. Caldwell, "Safe human robo interaction via energy regulation control," in Proc. IEEE/RSJ Int. Conf. Intell. Robots Syst, 2009, pp. 35-41.

[18] A. Albu-Schäffer, C. Ott, and G. Hirzinger, "A unified passivity-based control framework for position, torque and impedance control of flexible joint robots," Int. J. Robot. Res., vol. 26, no. 1, pp. 23-39, 2007.

[19] A. M. Zanchettin, B. Lacevic, and P. Rocco, "A novel passivity-based control law for safe human-robot coexistence," in Proc. 2012 IEEE/RSJ Int. Conf., Intell. Robots Syst. (IROS). IEEE, 2012, pp. 2276-2281.

[20] S. Stramigioli, "Energy-aware robotics," in Mathematical Control Theory I. Berlin, Germany: Springer, 2015, pp. 37-50. 
[21] F. Ferraguti et al., "An energy tank-based interactive control architecture for autonomous and teleoperated robotic surgery," IEEE Trans. Robot., vol. 31 , no. 5, pp. 1073-1088, Oct. 2015 .

[22] C. Schindlbeck and S. Haddadin, "Unified passivity-based cartesian force/impedance control for rigid and flexible joint robots via task-energy tanks," in Proc. 2015 IEEE Int. Conf. Robot. Autom., 2015, pp. 440-447.

[23] A. Dietrich, X. Wu, K. Bussmann, C. Ott, A. Albu-Schäffer, and S. Stramigioli, "Passive hierarchical impedance control via energy tanks," IEEE Robot. Autom. Lett., vol. 2, no. 2, pp. 522-529, Apr. 2017.

[24] B. Hannaford and J.-H. Ryu, "Time-domain passivity control of haptic interfaces," IEEE Trans. Robot. Autom., vol. 18, no. 1, pp. 1-10, Feb. 2002.

[25] J.-H. Ryu, C. Preusche, B. Hannaford, and G. Hirzinger, "Time domain passivity control with reference energy following," IEEE Trans. Control Syst. Technol., vol. 13, no. 5, pp. 737-742, Sep. 2005

[26] S. Stramigioli, C. Secchi, A. J. van der Schaft, and C. Fantuzzi, "Sampled data systems passivity and discrete port-Hamiltonian systems," IEEE Trans. Robot., vol. 21, no. 4, pp. 574-587, Aug. 2005.
[27] T. S. Tadele, T. de Vries, and S. Stramigioli, "PID motion control tuning rules in a damping injection framework," in Proc. Amer. Control Conf., 2013, pp. 4957-4962.

[28] N. Hogan, "Impedance control: An approach to manipulation," in Proc. Amer. Control Conf., 1984, pp. 304-313.

[29] S. Stramigioli, Modeling and IPC Control of Interactive Mechanical Systems a Coordinate Free Approach. Berlin, Germany: Springer, 2001.

[30] G. Schreiber, A. Stemmer, and R. Bischoff, "The fast research interface for the kuka lightweight robot," in Proc. 2010 IEEE Int. Conf. Robot. Autom., 2010, pp. 15-21.

[31] S. Chitta et al., "ros control: A generic and simple control framework for ros," J. Open Source Softw., vol. 2, 2017, Art. no. 456. [Online]. Available: http://www.theoj.org/joss-papers/joss.00456/10.21105.joss.00456.pdf

[32] B. Siciliano, L. Sciavicco, L. Villani, and G. Oriolo, Robotics: Modelling, Planning and Control (Advanced Textbooks in Control and Signal Processing). Berlin, Germany: Springer, 2009. 